\title{
The appropriate use of non-steroidal anti- inflammatory drugs in rheumatic disease: opinions of a multidisciplinary European expert panel
}

\author{
Gerd Burmester, ${ }^{1}$ Angel Lanas, ${ }^{2}$ Luigi Biasucci, ${ }^{3}$ Matthias Hermann, ${ }^{4}$ \\ Stefan Lohmander, ${ }^{5}$ Ignazio Olivieri, ${ }^{6}$ Carmelo Scarpignato, ${ }^{7}$ Josef Smolen, ${ }^{8}$ \\ Chris Hawkey, ${ }^{9}$ Adam Bajkowski, ${ }^{10}$ Francis Berenbaum, ${ }^{11}$ Ferdinand Breedveld, ${ }^{12}$ \\ Peter Dieleman, ${ }^{13}$ Maxime Dougados, ${ }^{14}$ Thomas MacDonald, ${ }^{15}$ Emilio Martin Mola, ${ }^{16}$ \\ Tony Mets, ${ }^{17}$ Nele Van Den Noortgate, ${ }^{18}$ Herman Stoevelaar ${ }^{19}$
}

\begin{abstract}
- Additional data (supplementary table and figures) are published online only. To view these files please visit the journal online at (http://ard.bmj.com).
\end{abstract}

For numbered affiliations see end of article.

\section{Correspondence to}

Professor Gerd R Burmester, Department of Rheumatology and Clinical Immunology, Charité - University Medicine, Free University and Humboldt University Berlin, Charitéplatz 1 10117, Berlin, Germany: gerd.burmester@charite.de

Accepted 18 July 2010 Published Online First 10 September 2010
This paper is freely available online under the BMJ Journals unlocked scheme, see http:// ard.bmj.com/info/unlocked.dtl

\begin{abstract}
Introduction Given the safety issues of nonsteroidal anti-inflammatory drugs (NSAID) and the robustness of guidelines, making treatment choices in daily clinical practice is increasingly difficult. This study aimed systematically to analyse the opinions of a multidisciplinary European expert panel on the appropriateness of different NSAID, with or without the use of a proton pump inhibitor (PPI), in individual patients with chronic rheumatic disease.

Methods Using the Research and Development/ University of California at Los Angeles appropriateness method, the appropriateness of five (non-)selective NSAID with or without a PPI was assessed for 144 hypothetical patient profiles, ie, unique combinations of cardiovascular and gastrointestinal risk factors. Appropriateness statements were calculated for all indications.
\end{abstract}

Results All options without PPI were considered appropriate in patients with no gastrointestinal/ cardiovascular risk factors. Cyclooxygenase-2 selective inhibitors (C2SI) alone and non-selective NSAID plus PPI were preferred for patients with elevated gastrointestinal risk and low cardiovascular risk. Naproxen plus PPI was favoured in patients with high cardiovascular risk. For the combination of high gastrointestinal/ high cardiovascular risk the use of any NSAID was discouraged; if needed, naproxen plus PPI or a C2SI plus PPI could be considered. Discussion The panel results may support treatment considerations at the level of individual patients, according to their gastrointestinal/cardiovascular risk profile.

Non-steroidal anti-inflammatory drugs (NSAID) belong to the mainstay treatments for chronic rheumatic diseases. Despite similar effectiveness, ${ }^{1-4}$ the currently available NSAID show pronounced differences in their safety profile. Classic NSAID carry a substantial risk of upper and lower gastrointestinal events, varying from mild symptoms to gastroduodenal ulcers and related serious complications. ${ }^{5}{ }^{6}$ Besides the dosage and frequency of NSAID use, several patient conditions have been identified to increase the risk of upper gastrointestinal complications, including advanced age, a history of gastrointestinal ulcer and concomitant treatment with corticosteroids, aspirin or anticoagulants. ${ }^{7}$ The later introduced cyclooxygenase- 2 selective inhibitors (C2SI) exhibit a more favourable gastrointestinal safety profile, ${ }^{8}$ albeit with individual differences. However, serious concerns about their cardiovascular toxicity have led to the market withdrawal of rofecoxib and regulatory warnings (European Medicines Agency) for the others. ${ }^{9}$ Following new reports that the increased cardiovascular risk may also apply to non-selective NSAID, the US Food and Drug Administration issued 'black box' safety warnings for the entire NSAID drug class. ${ }^{10}$ Consequently, the choice between the various NSAID is dominated by an uneasy application of the 'least harm principle', balancing the various potential adverse events. Despite the available recommendations, there are still several 'historical' myths that may perpetuate wrong habits and beliefs in clinical practice. ${ }^{11}$ In addition, guidelines are generally insufficiently detailed to support, at the level of the individual patient, a treatment choice that duly takes into account (combinations of) different cardiovascular and gastrointestinal risk factors. To bridge this gap between science and practice, we conducted a European panel study, combining the evidence from clinical studies with the opinions of experts from various disciplines.

\section{METHODS}

The Research and Development/University of California at Los Angeles (RAND/UCLA) appropriateness method ${ }^{12-14}$ was used.

\section{Panel composition}

The panel consisted of 18 experts from 10 European countries, representing all relevant disciplines (see appendix 1). Selection of experts was based on their specific expertise in the field of NSAID.

\section{Panel process}

The panel first met in January 2008 to set up the initial rating structure, ie, study population, treatment options (distinctive NSAID or NSAID groups) and clinical variables (relevant to the choice of different NSAID), see supplementary table 1, available online only.

Using an electronic rating program, panellists individually assessed the appropriateness of selected therapeutic options for a number of 
mutually exclusive profiles on a nine-point scale (reference values: 1, inappropriate; 5, uncertain; 9, appropriate). Following the RAND/UCLA definition, a treatment had to be considered appropriate if the expected benefits exceeded the potential negative consequences by a sufficient margin. ${ }^{12}$ Financial costs or other potential constraints had to be disregarded. Together with the rating program, the experts received a literature overview of which the scope and boundaries were determined during the first meeting. This (electronic) document was rather an extensive data overview than a comprehensive synthesis of clinical evidence, and reflected the published English-language literature from 1998 to 2008, with a focus on reports with the highest level of evidence.

The results of the first round were discussed during a plenary meeting (June 2008), leading to a revision of the rating structure and refinement of some treatment options and definitions (see box 1). Thereafter a second individual rating round took place, including 144 different patient profiles and 10 treatment options. Based on the median score and the extent of agreement between the panellists, appropriateness statements (appropriate, inappropriate, uncertain) were calculated for all indications, according to common RAND/UCLA rules. ${ }^{13}$ Indications were deemed appropriate if the median panel score was between 7 and 9, and inappropriate if the median was between 1 and 3, both without disagreement between panellists. Disagreement was defined as a situation in which at least six panellists scored in each of the sections 1-3 and 7-9. All indications not falling in the categories 'appropriate' and 'inappropriate' were labelled 'uncertain'.

A third panel meeting (November 2008) was organised to discuss the second round results and their applicability in daily clinical practice. The final ratings were embedded in an electronic tool that allows quick reference of the appropriateness statements for any of the patient profiles included.

\section{Statistical analysis}

A correlation matrix (using Spearman's correlation coefficient for ordinal variables) was applied to investigate the agreement between individual ratings, within and across the various specialities included.

\section{RESULTS}

\section{Panel results}

Of all theoretical indications rated in this study, approximately one-third were deemed inappropriate, largely attributable to classic NSAID without proton pump inhibitors (PPI) (see supplementary figure 1 , available online only). The percentage of appropriate indications was highest for naproxen plus PPI, followed by celecoxib alone and ibuprofen/diclofenac plus PPI. The outcomes were uncertain for more than half of the indications, of which approximately a quarter was caused by disagreement (strong opposite opinions), with highest values for naproxen plus PPI and celecoxib with or without PPI. Global figures show considerable variations in ratings over and within all specialties (supplementary figure 2, available online only).

For the patient profile without any unfavourable conditions, all NSAID without PPI (both classic and C2SI) were considered appropriate (supplementary figure 1, available online only). The presence of any gastrointestinal risk factors led to inappropriate outcomes of classic NSAID alone in the majority of cases. In patients with a low cardiovascular risk and a history of no or uncomplicated upper gastrointestinal events, celecoxib alone and classic NSAID plus PPI were generally favoured over the other options (figure 1). The combination of low cardiovascular risk and a history of complicated upper gastrointestinal events lowers the appropriateness of C2SI alone and naproxen plus PPI (uncertain). For patients with high cardiovascular risk, naproxen plus PPI showed the highest percentage of appropriate indications (46\%) (figure 1), whereas the other options scored between

\section{Box 1 Overview of treatment options, variables and definitions used in the second rating round}

- Patient population

- Patients with a chronic rheumatic disease for whom treatment with a NSAID is considered

- Treatment options

- Ibuprofen, diclofenac, naproxen, celecoxib, etoricoxib, all without or with the addition of a PPI

- Clinical variables used for the construction of patient profiles

- Age $(<65$ years; $\geq 65$ years $)$

- History of upper gastrointestinal events (none; uncomplicated; complicated)

- Uncomplicated: previous uncomplicated oesophageal, gastric or duodenal event (eg, significant symptoms, ulcer discovered by a clinically indicated work-up);

- Complicated: previous complicated oesophageal, gastric or duodenal event (bleeding, obstruction or perforation)

- Cardiovascular risk (low; high)

- 10-Year risk of fatal cardiovascular disease (heart attack, stroke), based on the (country-specific) HeartScore of the European Society of Cardiology; low: $<10 \%$; high: $\geq 10 \%$

- Use of low-dose aspirin (yes/no; only in patients with high cardiovascular risk)*

- Use of anticoagulants or other antiplatelet agents (yes/no)

- Anticoagulants: warfarin, acenocoumarol

- Antiplatelet agents: other than low-dose aspirin, for example clopidogrel (only in patients with high cardiovascular risk)*

- Use of systemic corticosteroids (yes/no)

- Treatment pattern (intermittent; continuous)

- Intermittent: short-term but repetitive

- Continuous: chronic use with standard doses

* It is assumed that patients with low cardiovascular risk do not take low-dose aspirin or other antiplatelet agents. 


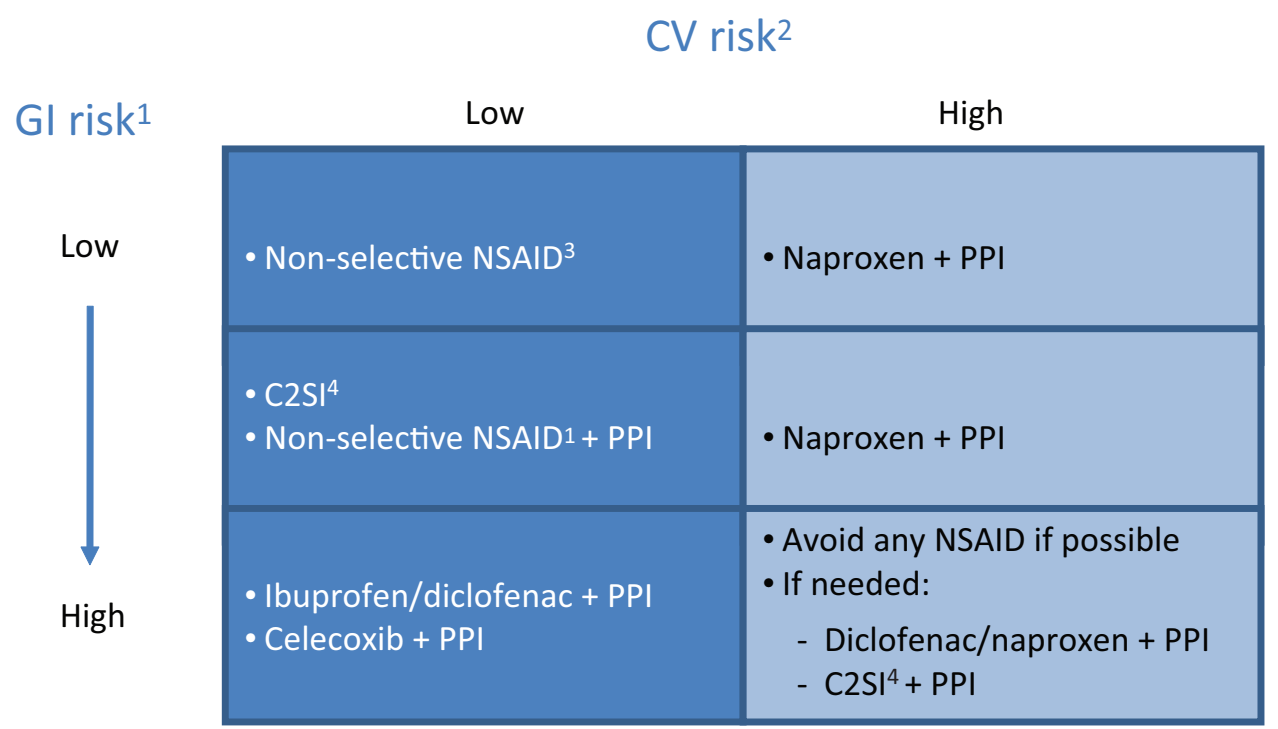

\footnotetext{
${ }^{1}$ Increasing GI risk is related to the number of GI risk factors that are present (previous upper Gl event, age $\geq 65$ years, continuous NSAID use, and concomitant use of aspirin/anticoagulants/corticosteroids).

${ }^{2}$ CV risk: 10 -year risk of fatal CV event (low < 10\%; high $\geq 10 \%$ ).

3 Ibuprofen/diclofenac/naproxen

${ }^{4}$ Celecoxib, etoricoxib
}

Figure 1 Global pattern of appropriate treatments in relation to gastrointestinal (GI) and cardiovascular (CV) risk levels. C2SI, cyclooxygenase-2 selective inhibitor; NSAID, non-steroidal anti-inflammatory drug; PPI, proton pump inhibitor.

$0 \%$ and $12 \%$. Appropriateness ratings for naproxen plus PPI showed a negative association with the severity of gastrointestinal risk factors and concomitant use of low-dose aspirin. In the highest risk category (high cardiovascular risk, on aspirin, history of complicated upper gastrointestinal events), none of the options was rated appropriate, whereas $56 \%$ of indications were deemed inappropriate (figure 1). Uncertain outcomes were largely the result of equivocal (ratings in section 4-6) or diffuse rating patterns.

Additional analyses showed that the agreement between panellists of the same speciality was not higher than that across specialities. The panel discussion after the second round revealed that much of the disagreement was related to different individual perceptions of the notion of appropriateness. The definition implies that the expected benefits should outweigh the potential negative consequences by 'a sufficient margin', but does not specify what constitutes 'sufficient'. Particularly for options with the addition of a PPI this led to divergent opinions for which no consensus could be reached. As financial costs had to be disregarded, some panellists considered a PPI as being always appropriate because they assumed that PPI carry a low potential for clinical harm. Others mentioned that they had rated the addition of a PPI only appropriate if they considered it a clear gain of gastrointestinal safety, pointing to a different risk avoidance attitude.

\section{Electronic tool}

Data were embedded in an electronic tool that allows the user to select a patient profile and to see the panel recommendations for that profile, including the distribution of panel ratings (figure 2). The program is accessible via http://www.e-hims.com/Sensar.

\section{DISCUSSION}

This study combines the insights and views from different perspectives to determine the appropriateness of different NSAID regimens in patients with chronic rheumatic disease. So far, two other studies on the same topic that used a similar approach have been published. However, the first study could be considered outdated because at the time of the study (2002), ${ }^{15}$ there were just a few indications of the cardiovascular risk of NSAID, causing the cardiovascular risk to influence the ratings minimally. The second, recently published, study by Chan et a $1^{16}$ had an international multidisciplinary panel that rated the appropriateness of six therapeutic regimens (nonnaproxen, naproxen, C2SI; all with and without PPI/misoprostol) for 288 clinical vignettes. Similarities in results with our study were first seen for the extremes of the patient population (with no cardiovascular and gastrointestinal risk factors at all: classic NSAID alone as appropriate; both a high cardiovascular and gastrointestinal risk: use of any NSAID is discouraged) and for those with low/average cardiovascular risk and high gastrointestinal risk (classic NSAID plus PPI or C2SI plus PPI). Differences were noted for the appropriateness of C2SI alone in patients with low cardiovascular risk and low/average gastrointestinal risk (being appropriate in our study vs uncertain). Divergence may partly be due to differences in methodology (eg, distinguishing between different members of the C2SI class in our study) and also in timing; our study was conducted 2 years after that of Chan et al, ${ }^{16}$ and in the meantime many new study reports became available. Knowledge progression, changing guidelines and increased expertise may influence treatment decisions, which emphasises the need for periodic updates of panel results, including ours. 
Appropriate use of (non-) selective NSAIDs in chronic rheumatic disease

PATIENT PROFILE

RECOMMENDATIONS

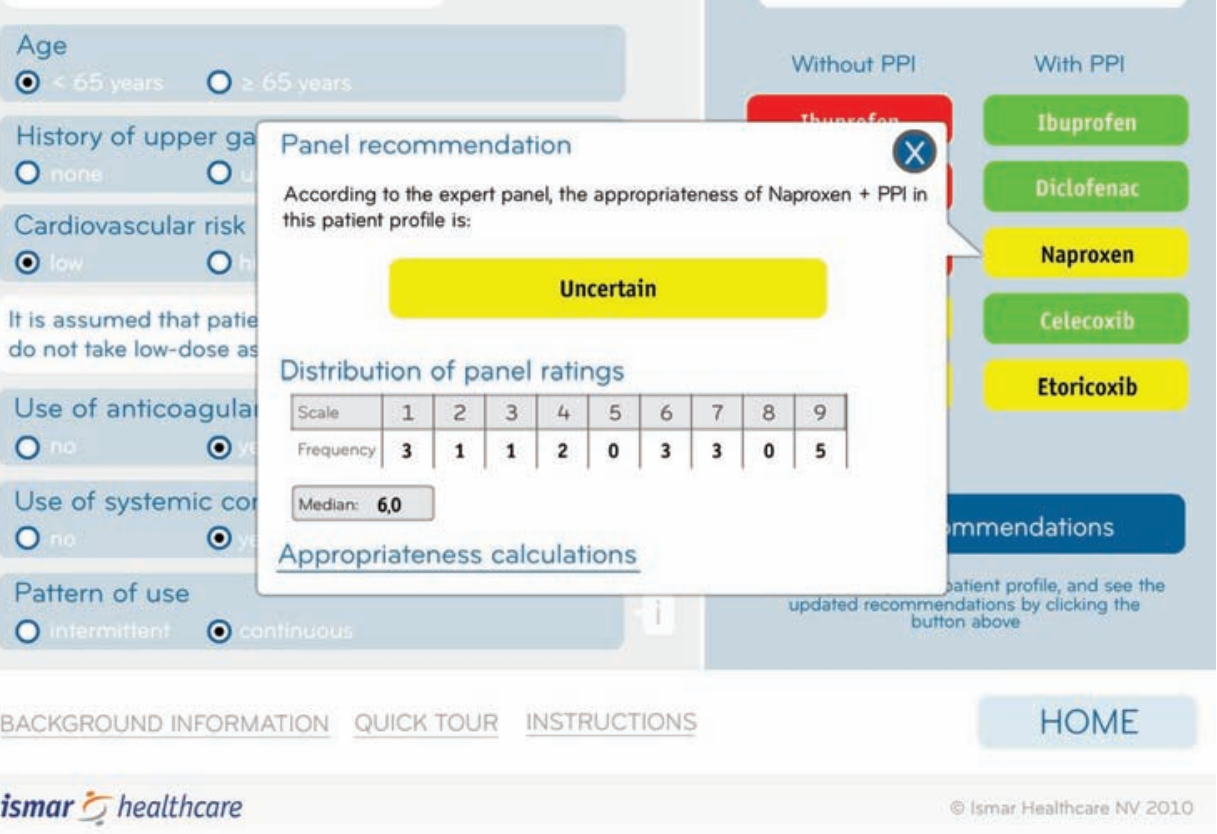

Figure 2 User interface of the electronic tool. NSAID, non-steroidal anti-inflammatory drug; PPI, proton pump inhibitor.

Given the complexity of data and to avoid the risk of oversimplification, we feel that an electronic tool (see figure 2) may offer the best opportunities as a quick reference guide to the panel results. In the complex and dynamic area of NSAID use, we feel that such an approach will offer the best chances of benefiting from the perspective of both science and practice.

Funding The study was supported by an unrestricted educational grant from Pfizer. The sponsor was not involved in the panel process and preparation of the manuscript.

Competing interests Panel members received honoraria from Pfizer for the rating activities and their participation in the panel meetings. GB has received fees for clinical trials from Pfizer and is a member of advisory boards and speakers bureau of Pfizer Germany and MSD Germany. AL has participated as a member of the steering committee of the CONDOR trial and as a speaker in symposiums sponsored by Pfizer. AL has also been advisor to AstraZeneca. LB has received fees for speeches and consultancy from Pfizer, Siemens diagnostic, Sanofi-Aventis and Merck. LB has also received a grant from Sanofi-Aventis. MH has received speaking fees from Pfizer and Novartis. SL has received honoraria as a member of advisory boards to Abbott, Boehringer, MerckSerono, Nic0x, Pfizer, Sanofi-Aventis. IO received consulting fees, speaking fees and/or honoraria from Schering-Plough, Bristol-Myers Squibb, Pfizer-Wyett, Abbott, Merck Pharmaceutical, Novartis, Sanofi-Aventis and Roche. CS is on the speakers bureau of Pfizer, AstraZeneca and SIDEM Pharma. His laboratory has received research grants from Pfizer. JS has received honoraria for advisory board activities from Pfizer and Novartis. HS has received honoraria from Pfizer for advice to the design of the study and data analysis. TMM has received research grants from Novartis, Pfizer, Ipsen \& Menarini; principal investigator for Pfizer, Novartis, Ipsen \& Menarini; received consulting or speakers fees from Pfizer, Novartis, Kaiser Permanente, Takeda, Recordati, Servier, Menarini, NiCox \& AstraZeneca. $\mathrm{CJH}$ received research funding and/or honoraria from AstraZeneca, Atlantic Pharmaceuticals, Bayer, Logical Therapeutics, Novartis Pharma, Pfizer and Reckitt Benckiser. FB received speaker honoraria from Abbott Laboratories. $T M, P D, F B, E M M, A B, M D, N V D N$ : none apart from the general statement in the article: "Panel members received honoraria from Pfizer for the rating activities and their participation in the panel meetings."

Provenance and peer review Not commissioned; externally peer reviewed.

Authors affiliations 'Department of Rheumatology and Clinical Immunology, Charité - University Medicine, Free University and Humboldt University Berlin, Berlin, Germany

2Department of Gastroenterology, University of Zaragoza, Zaragoza, Spain ${ }^{3}$ Institute of Cardiovascular, Medicine, Catholic University, Rome, Italy ${ }^{4}$ Department of Cardiology, University Hospital Zürich, Zürich, Switzerland
5Department of Clinical, Sciences, Lund University, Lund, Sweden

${ }^{6}$ Department of Rheumatology, Ospedale San Carlo, Potenza, Italy

${ }^{7}$ Department of Clinical Sciences, Division of Gastroenterology, University of Parma, Parma, Italy

${ }^{8}$ Department of Internal Medicine III, Division of Rheumatology, Medical University of Vienna, Vienna, Austria

${ }^{9}$ Nottingham Digestive Diseases Centre \& BRU, University Hospital, Nottingham, UK ${ }^{10}$ Bradshaw Medical Centre and Steering Committee representative of the Primary Care Rheumatology Society, Orrell, Wigan, UK

${ }^{11}$ Department of Rheumatology, University Pierre \& Marie Curie Paris VI, Saint-Antoine hospital, AP-HP, Paris, France

${ }^{12}$ Leiden University Medical Centre, Leiden, The Netherlands

${ }^{13}$ Department of General Medicine, University of Antwerp (ELIZA), Wilrijk, Belgium

${ }^{14}$ Paris-Descartes University, Medicine Faculty; UPRES EA 4058; AP-HP, Cochin Hospital, Rheumatology B Dept, Paris, France

${ }^{15}$ Medicines Monitoring Unit (MEMO), Division of Medical Sciences, University of Dundee, Ninewells Hospital and Medical School, Dundee, UK

${ }^{16}$ Department of Rheumatology, Hospital Universitario La Paz, Autonoma University Madrid, Madrid, Spain

${ }^{17}$ Department of Gerontology \& Geriatrics VUB, University Hospital, Brussels, Belgium

${ }^{18}$ Department of Geriatric Medicine, University Hospital Ghent, Ghent, Belgium

${ }^{19}$ Centre for Decision Analysis and Support, Ismar Healthcare, Lier, Belgium

\section{REFERENCES}

1. Hur C, Chan AT, Tramontano AC, et al. Coxibs versus combination NSAID and PPI therapy for chronic pain: an exploration of the risks, benefits, and costs. Ann Pharmacother 2006;40:1052-63.

2. Garner SE, Fidan DD, Frankish RR, et al. Celecoxib for rheumatoid arthritis. Cochrane Database Syst Rev 2002;4:CD003831.

3. Deeks JJ, Smith LA, Bradley MD. Efficacy, tolerability, and upper gastrointestinal safety of celecoxib for treatment of osteoarthritis and rheumatoid arthritis: systematic review of randomised controlled trials. BMJ 2002;325:619-23.

4. Richy F, Scarpignato C, Lanas A, et al. Efficacy and safety of piroxicam revisited. A global meta-analysis of randomised clinical trials. Pharmacol Res 2009;60:254-63.

5. Laine L. Proton pump inhibitor co-therapy with nonsteroidal anti-inflammatory drugs - nice or necessary? Rev Gastroenterol Disord 2004;4(Suppl 4):S33-41.

6. Schoenfeld P, Kimmey MB, Scheiman J, et al. Review article: nonsteroidal anti-inflammatory drug-associated gastrointestinal complications - guidelines for prevention and treatment. Aliment Pharmacol Ther 1999:13:1273-85.

7. Singh G. Gastrointestinal complications of prescription and over-the-counter nonsteroidal anti-inflammatory drugs: a view from the ARAMIS database. Arthritis, Rheumatism, and Aging Medical Information System. Am J Ther 2000;7:115-21. 
8. Rostom A, Muir K, Dubé C, et al. Gastrointestinal safety of cyclooxygenase-2 inhibitors: a Cochrane collaboration systematic review. Clin Gastroenterol Hepatol 2007;5:818-28.

9. European Medicines Agency (EMEA). Public statement, London 2005, EMEA/62838/2005. http://www.emea.europa.eu/pdfs/human/press/pr/6275705en. pdf (Accessed: July 2010).

10. US Food and Drug Administration. FDA news release, April 2005. http://www. fda.gov/NewsEvents/Newsroom/PressAnnouncements/2005/ucm108427.htm (Accessed: July 2010)

11. Hunt RH, Lanas A, Stichtenoth DO, et al. Myths and facts in the use of antiinflammatory drugs. Ann Med 2009;41:423-37.

12. Brook RH, Chassin MR, Fink $A$, et al. A method for the detailed assessment of the appropriateness of medical technologies. Int J Technol Assess Health Care 1986;2:53-63.

13. Fitch K, Bernstein SJ, Aguilar MS, et al. The RAND/UCLA appropriateness method user's manual. http://www.rand.org/pubs/monograph reports/MR1269 (Accessed: July 2010).

14. Shekelle P. The appropriateness method. Med Decis Making 2004;24:228-31.

15. Dubois RW, Melmed GY, Henning JM, et al. Guidelines for the appropriate use of non-steroidal anti-inflammatory drugs, cyclo-oxygenase-2-specific inhibitors and proton pump inhibitors in patients requiring chronic anti-inflammatory therapy. Aliment Pharmacol Ther 2004;19:197-208.
16. Chan FK, Abraham NS, Scheiman JM, et al. Management of patients on nonsteroidal anti-inflammatory drugs: a clinical practice recommendation from the First International Working Party on Gastrointestinal and Cardiovascular Effects of Nonsteroidal Anti-inflammatory Drugs and Anti-platelet Agents. Am J Gastroenterol 2008;103:2908-18.

\section{APPENDIX 1}

European expert panel on the appropriate use of NSAID Gerd R Burmester, Rheumatology, Germany; Francis Berenbaum, Rheumatology, France; Ferdinand Breedveld, Rheumatology, The Netherlands; Maxime Dougados, Rheumatology, France; Emilio Martín Mola, Rheumatology, Spain; Ignazio Olivieri, Rheumatology, Italy Josef Smolen, Rheumatology, Austria; Stefan Lohmander, Orthopaedics, Sweden Luigi M Biasucci, Cardiology, Italy; Matthias Hermann, Cardiology, Switzerland; Tom MacDonald, Cardiology and Clinical Pharmacology, UK; Chris Hawkey, Gastroenterology, UK; Angel Lanas, Gastroenterology, Spain; Carmelo Scarpignato, Gastroenterology and Clinical Pharmacology, Italy; Adam Bajkowski, Family Medicine UK; Peter Dieleman, Family Medicine, Belgium; Tony Mets, Geriatrics, Belgium; Nele Van Den Noortgate, Geriatrics, Belgium. 